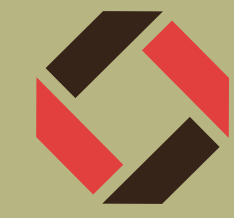

\title{
Spaced out: How astronaut headgear inspired personal radiation detection
}

\author{
By Omar Fabián
}

$\mathrm{H}$ ouston, Texas, 1969. It's the height of the space race, but Robert L. Fleischer's focus is elsewhere. Fleischera materials researcher from General Electric (GE) in Schenectady, N.Y.and several other scientists are gathered in a briefing room at the Manned Spacecraft Center (now the Lyndon B. Johnson Space Center). They'll be the first to study moon rocks up close upon the return of the Apollo 11 crew. Their mission isn't as glamorous as walking on the moon, but it is historical in its own right. For the time being, however, hearing about the rigors of collecting the precious rocks and the design specs of the Apollo spacesuit isn't quite enough to hold Fleischer's full attention. That is, until the speaker moves on to the topic of headgear and utters a five-letter word that immediately rouses his interest: Lexan.

A high-strength polycarbonate resin, Lexan is the lightweight plastic that forms the face of the iconic Apollo space helmet. But to Fleischer, it's much more than that. He's one of only a few people in the world who knows that Lexan is the perfect material for detecting cosmic rays. With a flash of insight - the kind that would dot his entire careerFleischer quickly made arrangements for his lab to study the space-faring helmets of the Apollo 8 crew (and later the Apollo 12 crew). In doing so, he and his team would launch the now common and potentially life-saving practice of measuring the amount of radiation ordinary people receive in their daily lives.
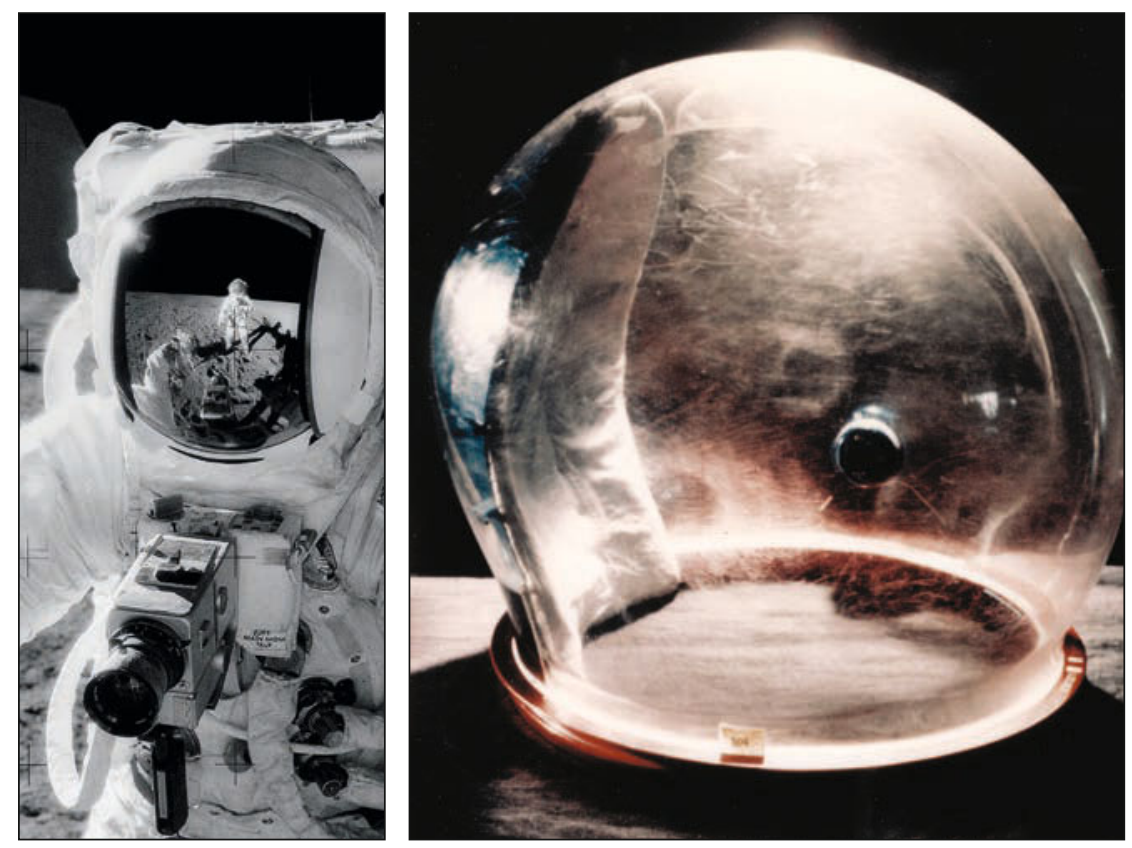

Left: Photo of Apollo 12 extravehicular activity. Alan L. Bean is shown collecting lunar soil samples, while Commander Charles Conrad Jr. is reflected in his helmet visor. Constructed by Air Lock, Inc., the pressure helmet consisted of a high-strength polycarbonate resin, Lexan. Credit: NASA. Right: Pressure helmet used by Fleischer to record cosmic rays. Credit: Robert L. Fleischer.

But that's not what he and his colleagues set out to do initially.

In the early 1960s, Fleischer was interested in recording cosmic rays, in collaboration with P. Buford Price and Robert M. Walker at GE. The team was motivated by recently published photographs of damage trails in mica formed naturally by fission fragments of uranium-235. These trails provided an indirect link to the past that complemented other radioactive dating techniques. On Earth, the density of trails imprinted in mica by the decay of natural uranium scales with age. On the Moon, the team reasoned, they should be able to recount the entire lunar history of cosmic ray bombardment, which produces similar radioactive fragments, via the observations of mica.

The then fledgling NASA was still years away from being able to provide the samples to prove their theory. In the meantime, the researchers had to make do with earthbound experiments. That would prove to be a blessing in disguise, because had they made one important realization sooner, their momentum would likely have fizzled: There is no mica on the Moon. The lunar landscape is too dry to form hydrous minerals such as mica and clay (even though ice has been found very recently). As Fleischer later admitted, "Luck is a precious ingredient in any work, and we have related how we benefited from ... ignorance about where mica is found."

None the wiser, the team pressed on.

They borrowed etching recipes from dislocation visualization studies to make particle tracks bigger and easier to observe. And they advanced from capturing tracks in crystalline solids to catching them in amorphous ones. Ultimately, they happened on an exceedingly good material for both creating and visualizing damage trails, one made in-house at GE. 
That's right, Lexan. The resin's uniform density and strong thermal insulation make the thermally induced distortions created by fission particles easy to spot.

Back to 1969 . It was arguably a spasm of relief, then, that stirred Fleischer from his seat at the space center. While his team would still have to wait to explore the lunar record of cosmic rays,
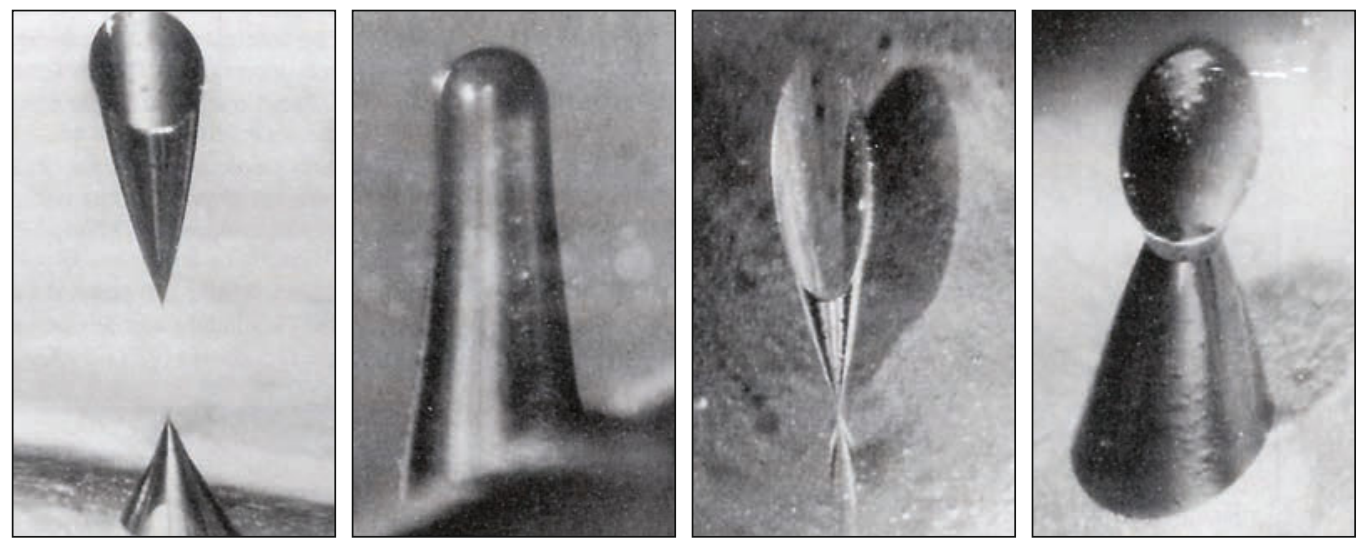

Etched tracks of cosmic rays that penetrated an Apollo 14 electrophoresis device that was made of Lexan. The taper and curvature of the tracks of the particles allow them to be individually identified. From left to right, argon, iron, calcium, and titanium ions. Credit: Springer Publishing Company. they at least had access to the first records of nuclear bombardment in space via the helmets. More importantly, those records were necessarily linked to the effects of radiation on the human body.

The team worked out that the amount of energy required by an alpha particle to produce a trail in Lexan is roughly the amount that would kill a $\mathrm{T}$ cell in the human kidney. Based on that metric, ionization by cosmic rays may have only damaged up to $0.000065 \%$ of brain cells in the Apollo crews. Though negligible for those missions, the team reported that the figure would extrapolate to $0.12 \%$ for periods of up to two years in space.
For the first time, Fleischer quipped, researchers had quantitative evidence of what was "going through the minds" of humans in space!

Even so, it isn't yet clear how cosmic rays affect the human nervous system. Some studies have linked them to chronic dementia in rodents. But the GE team's work was important, at the very least, in helping launch the study of ambient radiation effects, particularly those tied to radon. Modern instruments for measuring radon gas, the second-leading cause of lung cancer in the United States after cigarette smoke, are in fact particle track detectors. And, inspired by the radiation detector he serendipitously found in the form of the Apollo space helmet, Fleischer pursued the study of polycarbonate eyeglass lenses as an around-the-clock radon detector.

The versatility of particle track detection has since extended far beyond what the GE team could have imagined when they first set out to explore it. Some of the most notable applications have little or nothing to do with detecting cosmic rays. Shortly after the GE team was formed, for example, it was discovered that track etching could be used to engineer micropores for filtering cancer cells from human blood. The resulting patent on the process led to the spinoff Nuclepore Corporation.

Particle track detection highlights, in miniature (quite literally), the underemphasized virtues of cross-pollinating across different sects of science and engineering, a hallmark of materials science and engineering, in particular. It is no wonder then that the GE team members dedicated their compendium Nuclear Tracks in Solids to "those who have the vision of basic science in uncharted areas as one of the great forces that serve the long range good of mankind."
Left: Panel array leaning on the footpad of the lunar module on Apollo 16, which included General Electric experiments to capture cosmic ray tracks in Lexan and phosphate glass. Credit: NASA. Right: Robert L. Fleischer in his office at Union College. Items in the background include images of particle tracks, particle detector "book ends," and a mannequin with glasses representing the study of eye glasses. Credit: Elizabeth L. Fleischer. 